\title{
Novel observations in hereditary hemochromatosis: potential implications for clinical strategies
}

\author{
Dorine W. Swinkels ${ }^{1}$ and Robert E. Fleming ${ }^{2}$
}

${ }^{1}$ Radboud University Nijmegen Medical Centre, Nijmegen, The Netherlands; ${ }^{2}$ Saint Louis University School of Medicine, St. Louis, USA; E-mail:d.swinkels@labgk.umcn.nl doi:10.3324/haematol.2011.042036

(Related Original Articles on pages 500 and 507)

$\mathrm{R}$ emarkable advances in understanding the pathogenesis of hereditary hemochromatosis have been made since 1996, the year in which the HFE gene, which is responsible for the vast majority of cases, was discovered. ${ }^{1}$ The recognition of the central role of the iron regulatory hormone hepcidin in the pathogenesis of hereditary hemochromatosis has been particularly significant. In addition to HFE, defects in four additional genes have been found to cause hereditary hemochromatosis: hepcidin, transferrin receptor 2 (TFR2), hemojuvelin $(H J V)$, and ferroportin. ${ }^{2}$ Interestingly, these genes each encode for a protein that affects pathways influencing liver hepcidin synthesis or its interaction with the cellular iron export protein ferroportin. It is now clear that inadequate production of hepcidin or response to it is responsible for the excess dietary iron absorption and excess iron release from macrophages stores, and consequent hyperferremia, which characterize the classical hereditary hemochromatosis phenotype. For clinicians, the challenge is to diagnose hereditary hemochromatosis before this hyperferremia leads to irreversible tissue damage. At the same time the clinician must have ways to distinguish progressive heritable forms of iron overload from increasingly common acquired diseases with only moderately increased iron body stores, such as the metabolic syndrome. This challenge has raised interest in the possibility that recent bench top discoveries might lead to novel bedside laboratory tests. Two publications in this issue of Haematologica are relevant to this possibility. ${ }^{3,4}$ In one study, serum hepcidin levels were used to characterize the response of patients with HFE- or TFR2-associated hereditary hemochromatosis to an oral iron challenge. ${ }^{3}$ The other study identifed several clinically significant orphan mutations in the HFE gene. ${ }^{4}$ The contribution of each of these studies to a better understanding of the pathogenesis of hereditary hemochromatosis and the possible ways they might influence the future diagnostic work-up and monitoring of iron overload disorders are discussed.

\section{The relation between iron status and hepcidin levels in patients with HFE or TFR2 mutations}

While there are many aspects of the regulation of hepcidin expression by iron status that remain to be dissected, several lines of evidence indicate that liver iron stores and circulating ferri-transferrin ( $\mathrm{Tf}-\mathrm{Fe}_{2}$ ) each provide a signal to influence hepatocyte hepcidin expression (Figure 1). ${ }^{5}$ An increase in liver iron stores signals to hepcidin via certain members of the bone morphogenetic protein (BMP) family, particularly BMP6, in an autocrine and/or paracrine fashion. These extracellular signaling molecules act on hepatocellular BMP receptors to activate the intracellular SMAD signaling pathway and increase hepcidin transcription. Hemojuvelin is a BMP-coreceptor which augments the BMP signal. ${ }^{6}$ Circulating transferrin appears to be sensed via a hepatocellular complex which includes TFR1, TFR2 and HFE. ${ }^{5}$ Defects in HFE and TFR2 lead to relatively attenuated hepatocellular signaling to hepcidin via the MAPK and/or BMP/SMAD pathways resulting in decreased circulating hepcidin levels. ${ }^{5,-9}$ In this issue of Haematologica, Girelli et $a l^{3}$ describe their observation of a blunted serum hepcidin response upon an oral iron challenge in overloaded HFE C282Y homozygotes, confirming their previous findings using urine hepcidin as a read out. ${ }^{10}$ This observation is consistent



Figure 1. Putative iron regulatory pathways of hepatocyte hepcidin synthesis. This proposed model depicts two iron signals to hepcidin, one mediated by intracellular iron stores $(\mathrm{Fe})$ and the other by ferri-transferrin ( $\left(\mathrm{Tf}^{-} \mathrm{Fe}_{2}\right.$ ). Hepatocellular iron stores increase the expression of bone morphogenetic protein 6 (BMP6), which serves as an autocrine factor by interacting with surface BMP receptors. Hemojuvelin (HJV) is a BMP co-receptor which augments BMP binding. The consequent activation of intracellular SMAD proteins transduces a signal to increase hepcidin transcription. Under low iron conditions matriptase-2 (scissors) cleaves HJV from the cell surface, weakening the BMP6 signal. Extracellular $\mathrm{Tf}-\mathrm{Fe}_{2}$ mediates a second iron signal. In this schema $\mathrm{Tf}-\mathrm{Fe}_{2}$ displaces HFE from TfR1. HFE is then freed to interact with transferrin receptor 2 (TfR2). The HFE-TfR2 complex activates hepcidin transcription via MAPK and/or BMP/SMAD signaling. These pathways have recently been reviewed. ${ }^{5}$ 


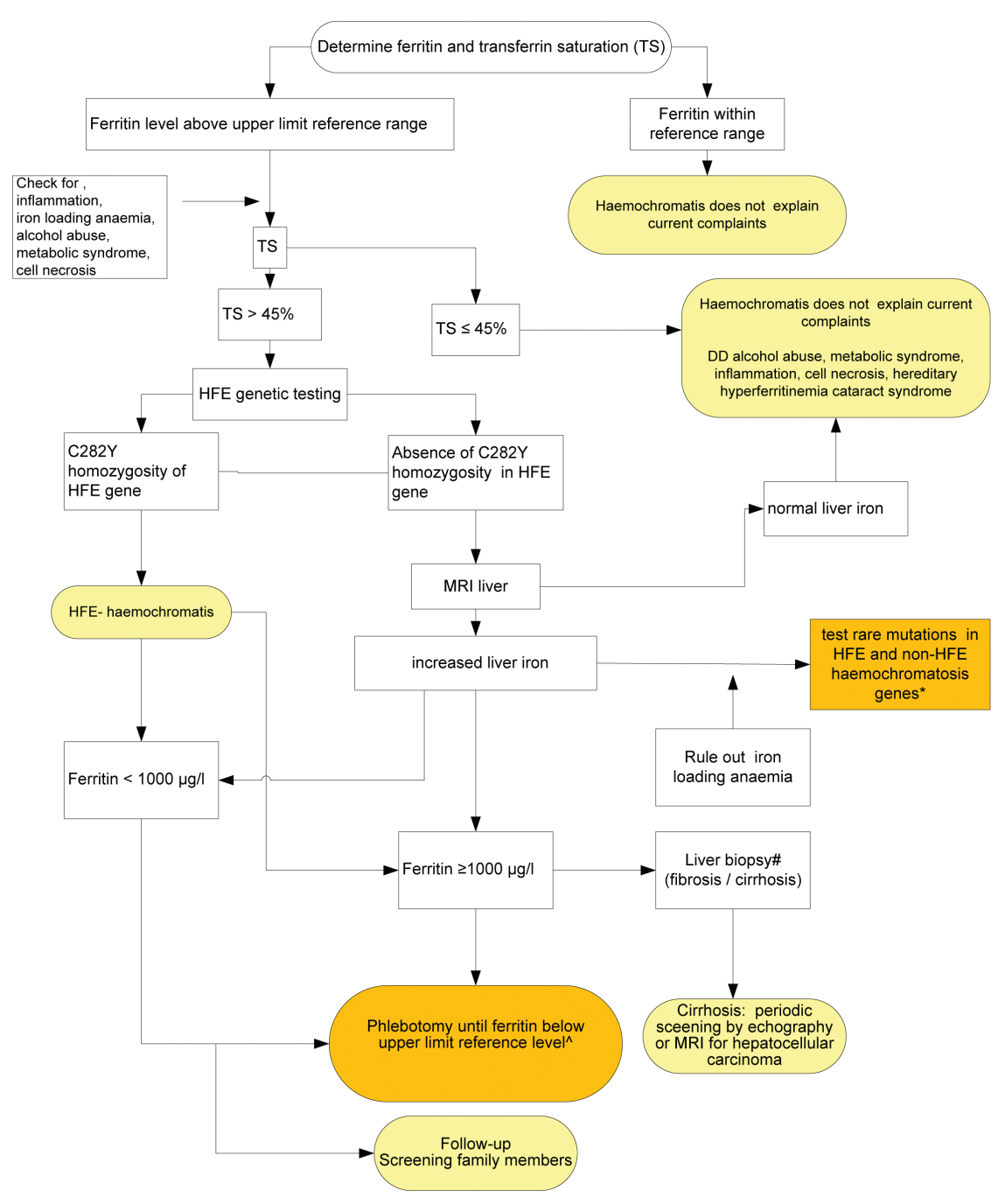

Figure 2. Proposed diagnostic and therapeutic algorithm for patients with suspected hemochromatosis. Orange boxes indicate the parts of the guideline for which the novel observations of the two publications in this issue might provide opportunities for improvement. * in case of heterozygosity for the $\mathrm{C282Y}$ mutations start searching for rare mutations in HFE in the other allele. Serum hepcidin/ferritin ratio may contribute to prioritization of order of gene investigations. If mutations are present the work-up is similar to that for HFE hemochromatosis with respect to phlebotomy, screening relatives, and periodic screening of the liver in the case of liver cirrhosis. ^ Low ferritin targets may lead to excessive iron absorption. Measurement of hepcidin could have a role in monitoring phlebotomy and to assess the ferritin target and frequency of phlebotomy. Figure adapted from van Bokhoven et al. ${ }^{15}$

with the concept that iron signaling to hepcidin is attenuated in the absence of HFE or TFR2. The inability to manipulate transferrin saturation in the TFR2-mutated patients, and the lack of specific measurement of differic transferrin levels do, however, make it difficult to use these studies to further substantiate the case that HFE and TFR2 act by sensing circulating differric transferrin.

The iron-depleted HFE C282Y patients had very low hepcidin levels, reflecting the combined effects of the HFE mutation and phlebotomy on hepcidin expression. These patients demonstrated an exaggerated increase in serum iron levels upon iron loading, corroborating previous reports directly measuring iron absorption. ${ }^{11}$ These observations draw further attention to the concern that lowering hepcidin levels by phlebotomy in patients with hereditary hemochromatosis may exacerbate the excess release of iron into the circulation with an ensuing vicious circle with such patients requiring more frequent maintenance phlebotomies. ${ }^{8,10}$ Interestingly, oral iron loading did not cause an exaggerated increase in serum iron levels in the phlebotomized TFR2 patients and the untreated HFE patients, despite their decreased hepcidin levels. These observations suggest that dietary iron absorption was not increased in these patients, and raises the possibility that compensatory mechanisms exist to partially offset the hepcidin-mediated effects on dietary iron absorption in hereditary hemochromatosis patients with elevated transferrin saturation. Alternatively, the excess iron absorbed from the diet in these hereditary hemochromatosis patients may have entered the circulation as non-transferrin bound iron which was rapidly cleared by the liver, and thus not reflected as a change in systemic serum iron levels.

\section{Novel HFE mutations associated with hereditary hemochromatosis}

By far the most common genetic cause of hereditary hemochromatosis is the C282Y mutation in HFE. ${ }^{1}$ The consequent loss of a critical disulfide bond results in a loss of HFE interaction with $\beta 2$-microglobulin and decreased trafficking of the HFE protein to the cell surface..$^{12}$ A more common, though less consequential HFE mutation leads to a substitution of histidine for aspartate at nucleotide 63 $(63 \mathrm{H} \rightarrow \mathrm{D}){ }^{1}$ The clinical implications of this mutation have been an area of controversy. Several other allelic variants have been reported, some of which of unknown significance. ${ }^{13}$ In this issue of the journal, Aguilar-Martinez et al. 
describe three novel HFE mutations which, as compound heterozygotes with the C282Y mutation, can lead to hemochromatosis. ${ }^{4}$ Knowledge of mutations that functionally affect $H F E$ is important in currently recommended strategies for the diagnostic work-up of the patient suspected of having hereditary hemochromatosis. ${ }^{14-16}$

\section{Diagnostic and monitoring strategies in hereditary hemochromatosis}

Unfortunately the early clinical signs and symptoms of iron overload are both common and non-specific. At the same time, the clinical penetrance of HFE mutations is quite low, making population screening unjustified. A high index of clinical suspicion is, therefore, essential for early diagnosis. In recently developed evidence-based guidelines physicians are recommended to consider testing for the disease in patients who have had unexplained symptoms consistent with iron toxicity for several months. The combined measurement of serum iron concentration, transferrin or total iron binding capacity (and calculation of transferrin saturation) and ferritin concentration provide a simple and reliable first assessment of body iron levels. HFE-gene testing is needed only in those cases with increased transferrin saturation and ferritin and when acquired causes of hyperferritinemia have been excluded (Figure 2). ${ }^{14-16}$ If the patient is homozygous for HFE C282Y, the diagnosis of HFE hemochromatosis can be established. If serum iron parameters are elevated but the pathognomonic HFE genotype (C282Y homozygous) is absent, determination of hepatic iron concentration is warranted. Magnetic resonance imaging is increasingly recognized as a useful non-invasive approach for this measurement, ${ }^{17}$ provided that proper software and calibration techniques are used, which is often problematic. In the selected cases with documented hepatic iron overload, a search for the non-classical genetic defects is then indicated (Figure 2). In their paper Aguilar-Martinez et al. ${ }^{4}$ suggest first testing for rare HFE mutations in HFE C282Y heterozygous patients. In the case that $\mathrm{C} 282 \mathrm{Y}$ mutations are absent, the priority order of the genes to be investigated might be directed by clinical, laboratory and pathological features and the local prevalence of certain gene defects. For instance in the case of presentation at young age, certain genes (HJV and hepcidin) might be checked first, and in the south-west of the Netherlands the $\mathrm{N} 144 \mathrm{H}$ mutation in the ferroportin gene might be the primary defect to look for. ${ }^{18}$ Ongoing improvements in genomic techniques and their automation and interpretation will further assist in efficient gene diagnosis ${ }^{19}$ and might preclude the need for prioritization, undoubtedly affecting future guidelines.

The measurement of serum hepcidin may also contribute to the diagnostic and therapeutic monitoring algorithms in patients with hereditary hemochromatosis. It is still too early to determine at this stage the role of hepcidin measurements in clinical practice; however, several comments on this issue can be made. First, measurement of hepcidin early in the diagnostic scheme cannot be currently recommended, as testing for the HFE C282Y mutation is straightforward and the majority of cases will be found to have this mutation. Second, for patients who do not have $\mathrm{C} 282 \mathrm{Y}$ homozygosity but do have documented iron overload, the hepcidin to ferritin ratio may be helpful in the prioritization of the genes to be investigated, i.e. a low ratio points towards defects in $H J V$ and hepcidin genes (Figure 2). Third, hepcidin could have a role in monitoring phlebotomy and assessing the ferritin target and frequency of phlebotomy (Figure 2). Fourth, the differences in hepcidin results obtained by enzyme-linked immunosorbent assays and mass-spectrometry methods, observed by Girelli et al. in their study, show that before hepcidin measurements can be used clinically, they need to be better standardized. ${ }^{3,20}$ Results and recommendations from a recent comparison of hepcidin levels from identical samples performed in different laboratories (the so-called hepcidin Round Robin 2) are expected in the course of 2011. We expect that this effort will ultimately aid in the definition of clinical decision limits that can be broadly applied, regardless of the technique or laboratory. Finally, as with most other hormones, hepcidin values in patients suspected of having hereditary hemochromatosis should only be interpreted as (in)appropriate in the context of other indices of iron metabolism.

\section{Concluding remarks}

The two articles in this issue of Haematologica provide further evidence that rare HFE mutations may account for body iron overload in C282Y heterozygous patients and that iron depletion by phlebotomy lowers hepcidin levels and increases iron absorption. These observations may be considerations in future evidence-based clinical guidelines for patients suspected of having hereditary hemochromatosis and inspire further studies on the role of serum hepcidin measurements in the clinical care of these patients.

Dorine W. Swinkels, M.D., Ph.D. is Professor of Experimental Clinical Chemistry at Radboud University Nijmegen Medical Center, Nijmegen, the Netherlands. Her work includes translation of novel factors that mediate iron homeostasis into new diagnostic assays and therapeutic strategies that can be implemented in the clinic. Her team's most recent achievements include the development of mass spectrometry and immunochemical assays for hepcidin that are currently the subject of several translational studies (www.hepcidinanalysis.com). Robert E. Fleming, M.D. is Professor of Pediatrics and Biochemistry \& Molecular Biology at Saint Louis University School of Medicine, St. Louis, MO, USA. His research interests include the molecular mechanisms by which iron influences hepcidin expression utilizing murine model systems.

Financial and other disclosures provided by the author using the ICMJE (www.icmje.org) Uniform Format for Disclosure of Competing Interests are available with the full text of this paper at www. haematologica.org.

\section{References}

1. Feder JN, Gnirke A, Thomas W, Tsuchihashi Z, Ruddy DA, Basava A, et al. A novel MHC class I-like gene is mutated in patients with hereditary haemochromatosis. Nat Genet. 1996;13(4):399-408.

2. Swinkels DW, Janssen MC, Bergmans J, Marx JJ. Hereditary hemochromatosis: genetic complexity and new diagnostic approaches. Clin Chem. 2006;52(6):950-68.

3. Girelli D, Trombini P, Busti F, Campostrini N, Sandri M, Pelucchi S, et al. A time course of hepcidin response to iron challenge in patients with HFE and TFR2 hemochromatosis. Haematologica 2011;96(4): 500-6.

4. Aguilar-Martinez P, Grandchamp B, Cunat S, Cadet E, Blanc F, Nourrit $\mathrm{M}$, et al. Iron overload in HFE C282Y heterozygotes at first genetic testing: a strategy for identifying rare HFE variants. Haematologica 2011;96(4):507-14. 
5. Hentze MW, Muckenthaler MU, Galy B, Camaschella C. Two to tango: regulation of mammalian iron metabolism. Cell. 2010;142(1): 24-38.

6. Babitt JL, Huang FW, Wrighting DM, Xia Y, Sidis Y, Samad TA, et al. Bone morphogenetic protein signaling by hemojuvelin regulates hepcidin expression. Nat Genet. 2006;38(5):531-9.

7. Nemeth E, Roetto A, Garozzo G, Ganz T, Camaschella C. Hepcidin is decreased in TFR2 hemochromatosis. Blood. 2005;105(4):1803-6.

8. van Dijk BA, Laarakkers CM, Klaver SM, Jacobs EM, van Tits LJ, Janssen MC, Swinkels DW. Serum hepcidin levels are innately low in HFE-related haemochromatosis but differ between C282Y-homozygotes with elevated and normal ferritin levels. Br J Haematol. 2008;142(6):979-85.

9. Tjalsma H, Laarakkers CMM, van Swelm RPL, Theurl M, Theurl I, Kemna EH, et al. Mass spectrometry analysis of hepcidin peptides in experimental mouse models. PLoS ONE 2011;6:e16762.

10. Piperno A, Girelli D, Nemeth E, Trombini P, Bozzini C, Poggiali E, et al. Blunted hepcidin response to oral iron challenge in HFE-related hemochromatosis. Blood. 2007;110(12):4096-100.

11. Roe MA, Collings R, Dainty JR, Swinkels DW, Fairweather-Tait SJ. Plasma hepcidin concentrations significantly predict interindividual variation in iron absorption in healthy men. Am J Clin Nutr. 2009;89(4):1088-91.

12. Waheed A, Parkkila S, Zhou XY, Tomatsu S, Tsuchihashi Z, Feder JN, et al. Hereditary hemochromatosis: effects of C282Y and H63D muta- tions on association with beta2-microglobulin, intracellular processing and cell surface expression of the HFE protein in COS-7 cells. Proc Nat Acad Sci USA. 1997;94(23):12384-9.

13. Lee PL, Beutler E. Regulation of hepcidin and iron-overload disease. Annu Rev Pathol. 2009;4:489-515.

14. European Association For The Study Of The Liver. EASL clinical practice guidelines for HFE hemochromatosis. J Hepatol. 2010;53(1):3-22.

15. van Bokhoven MA, van Deursen CT, Swinkels DW. Diagnosis and management of hereditary haemochromatosis. BMJ. 2011;342:c7251.

16. Swinkels DW, Jorna AT, Raymakers RA. Synopsis of the Dutch multidisciplinary guideline for the diagnosis and treatment of hereditary haemochromatosis. Neth J Med. 2007;65(11):452-5.

17. Fischer R, Harmatz PR. Non-invasive assessment of tissue iron over load. Hematology Am Soc Hematol Educ Program. 2009;215-21.

18. Njajou OT, Vaessen N, Joosse M, Berghuis B, van Dongen JW, Breuning $\mathrm{MH}$, et al. A mutation in SLC11A3 is associated with autosomal dominant hemochromatosis. Nat Genet. 2001;28(3):213-4.

19. Kuhlenbaumer G, Hullmann J, Appenzellerm S. Novel genomic techniques open new avenues in the analysis of monogenic disorders. Hum Mutat. 2010 Nov 18.

20. Kroot JJ, Kemna EH, Bansal SS, Busbridge M, Campostrini N, Girelli D, et al. Results of the first international round robin for the quantification of urinary and plasma hepcidin assays: need for standardization. Haematologica. 2009;94(12):1748-52.

\title{
The rationale for using hydroxycarbamide in the treatment of sickle cell disease
}

\section{David C. Rees}

\author{
King's College Hospital NHS Foundation Trust, Denmark Hill, London, UK; E-mail: david.rees@kcl.ac.uk \\ doi:10.3324/haematol.2011.041988
}

(Related Original Article on pages 526 and 534)

T he modern management of sickle cell disease (SCD) is based on three therapeutic approaches: blood transfusion (first used successfully in 1818), penicillin (discovered in 1928) and hydroxycarbamide (first synthesized in 1869). ${ }^{1}$ Dresler and Stein made this simple molecule from hydroxylamine, hydrochloric acid and potassium cyanide as a technical exercise in organic chemistry, as part of a series of experiments generating derivatives of urea. Hydroxycarbamide lay dormant for more than fifty years until it was studied as part of an investigation into the toxicity of protein metabolites and found to produce a megaloblastic anemia, which was thought to mimic pernicious anemia. ${ }^{2}$ In the early 1960s further in vitro studies showed that hydroxycarbamide had activity against leukemia cell lines and some tumors ${ }^{3}$ and this led to clinical studies showing particular activity in myleoproliferative disorders. ${ }^{4}$

Increased fetal hemoglobin $(\mathrm{HbF})$ production has long been recognized as one of the key factors which can ameliorate $\mathrm{SCD}^{5}$ and in the 1970s 5-azacytidine was investigated as an $\mathrm{HbF}$ promoting agent because of its potential ability to reactivate silenced $\gamma$-globin genes by inhibiting the methylation of deoxycytidine. Although 5-azacytidine successfully increased HbF levels in baboons, and subsequently in patients with SCD and thalassemia, it was relatively toxic. ${ }^{6}$ Hydroxycarbamide was also used in the early baboon experiments, partly as a cytotoxic control because it was known to have no effect on methylation and, perhaps surprisingly, was also found to promote $\mathrm{HbF}$ synthesis. Because of concerns about the toxicity of 5-azacytidine, hydroxycarbamide was developed as a safer alternative and an initial study in 2 adults with sickle cell anemia
(HbSS) (SCA) showed significant increases in both $\mathrm{HbF}$ and total hemoglobin.? Further observational studies followed, before the Multicenter Study of Hydroxyurea (MSH) study was published in 1995. In this double-blind randomized controlled study, 152 adult patients with SCA were assigned to hydroxycarbamide and 147 given placebo; the hydroxycarbamide group showed reductions in the rate of acute pain (median 2.5 vs. 4.5 episodes per year, $P<0.001$ ), acute chest syndrome $(25 \mathrm{vs} .51, P<0.001)$ and blood transfusion (48 vs. 73, $P<0.001){ }^{8}$ The study was stopped early because of the reduction in acute pain in the hydroxycarbamide arm. The only other published randomized controlled study involved a single-blind crossover study of 25 children and young adults with SCA treated for six months with hydroxycarbamide and for six months with placebo; hydroxycarbamide showed a treatment effect on reducing the number of hospitalizations $(P=0.0016)$ and total days in hospital $(P=0.0027) .{ }^{9}$ Since then a steady stream of registry, observational and follow-up studies have followed, all showing similar beneficial effects with increases in $\mathrm{HbF}$ levels and reductions in some acute complications. Most notably, two observational studies have suggested increased survival associated with long-term hydroxycarbamide use. A follow-up study of the patients in the original MSH study showed a $40 \%$ reduction in mortality in those who chose to continue hydroxycarbamide after nine years $(P=0.04) ;{ }^{10}$ a non-randomized study of patients in Greece with SCA, HbS/ $\beta^{0}$ thalassemia and $\mathrm{HbS} / \beta^{+}$thalassemia showed the probability of 10 -year survival was $86 \%$ for those taking hydroxycarbamide and $68 \%$ for those not taking it $(P=0.001){ }^{11}$ 